\title{
Pronóstico de las acciones vencidas esperadas para el periodo $t+1$, en el contexto del sistema de gestión de calidad para una empresa española del sector eléctrico con operaciones en México
}

Over-dead waited actions' forecast for period $t+1$, at the quality management system in an electric sector Spanish enterprise with operations in central Mexico

Abigail C. Tapia-Rodríguez, ${ }^{a,}$, Héctor D. Molina-Ruiz ${ }^{b}$

\begin{abstract}
:
Quality management system within the organizations allows quality levels' control in product or service scopes. Through the implementation of improvement actions, customer satisfaction can be increased. However, when these actions are not fulfilled, quality management system is affected having a barrier to reach high quality levels, which impacts customer satisfaction. It is pertinent, apply tools that, allow us to visualize quality management system's status. In present document, a statistical analysis is carried out to forecast period $t+1$, in expired quality actions field, in order to provide guidelines for decision makers, to support quality management system's use.
\end{abstract}

Keywords:

Corrective actions, Electric transformers, Quality management system

\section{Resumen:}

El sistema de gestión de calidad al interior de lar organizaciones, permite el control de los niveles de calidad del producto o servicio. A través de la puesta en marcha de acciones de mejora, la satisfacción del cliente se ve incrementada. Sin embargo, cuando dichas acciones no se llevan a término, el sistema de gestión de calidad, se ve mermado en la entrega de niveles elevados de calidad, lo cual impacta la satisfacción del cliente. Es pertinente, contar con la aplicación de herramientas que permitan vislumbrar el estado que guarda el sistema de gestión de calidad. En el presente documento, se realiza un análisis estadístico y se pronostica el periodo $t+1$, para las acciones de calidad vencidas, con objeto de brindar pautas a los tomadores de decisiones, para apoyar el uso del sistema de gestión de calidad.

\section{Palabras Clave:}

Acciones correctivas, Sistema de gestión de calidad, Transformadores eléctricos

\section{Introducción}

El sistema de gestión de calidad, permite la puesta en marcha de acciones o iniciativas de mejora, con las cuales, la organización incrementa sus niveles de calidad y la satisfacción del cliente. En este contexto, al tener acciones no cerradas (no finiquitadas, no terminadas), el nivel de calidad se ve mermado, por lo cual es importante generar información que permita forjar acciones y posibilite la toma de decisiones.

En el documento que se desarrolla a continuación, se plantea el contexto de la empresa objeto de estudio, así como el sistema de gestión de calidad. Por otro lado, se realiza la caracterización de los datos, referentes a las acciones vencidas del sistema de gestión de calidad (aquellas que no se han finiquitado), además se realiza el cálculo del índice de correlación y el pronóstico del periodo $t+1$, mediante el uso de mínimos cuadrados ordinarios (MCO), que permiten generar un panorama útil, con objeto de posibilitar el análisis de las acciones dentro de la organización.

\section{Desarrollo}

La empresa objeto de estudio de origen español encargada del diseño, fabricación, integración y comercialización de productos y soluciones para la 
medición, protección, automatización, control y comunicación en redes eléctricas. Esta empresa, además, es gestora de proyecto para la generación, transmisión y distribución de electricidad; dando cumplimiento a la certificación integral de las normas ISO 9001:2015 e ISO 14001: 2015. Cabe hacer mención que la empresa se encuentra en vías de certificación en la norma ISO 45001:2018.

Se tienen 10 procesos definidos desde el corporativo de la empresa objeto de estudio: marketing y ventas, proyectos y servicios, operaciones, I+D, compras, financiamiento y tesorería, tecnologías de la información, planificación y gestión, excelencia y recursos humanos.

Los tipos de transformadores son diseñados y fabricados con base en la aplicación, que pueden ser usados desde elementos de reducción de tensión hasta en la transformación de tensión en una central termoeléctrica. Dentro de los procesos de la organización se encuentra el sub-proceso de calidad el cual está dividido en:

- $\quad$ Sistema de Gestión de calidad (SGC)

- $\quad$ Calidad de Planta

- $\quad$ Calidad de proveedores

Uno de los procesos que intervienen en el tratamiento de los defectos y rechazos, es calidad de planta, ya que es responsables de realizar los análisis correspondientes por la mala calidad de los transformadores y el tratamiento que le corresponde según sea el problema detectado, todo esto ayudándose en la "norma de cohesión", la cual direcciona el tratamiento y codificación para cada uno de los trabajos a realizar, según el tipo de defecto y/o rechazo.

\subsection{Contexto teórico}

La calidad de los productos afecta la eficiencia de la producción, dado que los productos con baja calidad pueden desestabilizar las ventas, además de producir desperdicio de materiales y aumentar el costo financiero (Wang, Chen, Quiao \& Snoussi, 2018).

La identificación y el cálculo de los costos de calidad es uno de las bases para mejorar los procesos. Los costos de calidad no son exclusivamente una medida absoluta del desempeño, su importancia radica en que indica donde será más provechosa una acción correctiva para la empresa, y se utilizan como indicadores de áreas de la empresa susceptibles de mejora en sus productos y procesos (Gómez Alfonso, 2012).

Arredondo-González (2015) menciona que, la fase de control de los costos, ayuda a identificar las fallas operativas o incluso administrativas de la gestión que se está realizando dentro de la organización.

Los costos de la calidad son aquellos que no existirían si todas las actividades son necesarias o críticas, se hicieran siempre bien a la primera.

Los mismos se clasifican en:

- Costos de conformidad

\section{- $\quad$ Costos de no conformidad}

Cabe hacer mención que, los costos de no conformidad tienen diferentes raíces $y$, por tanto, diferentes tratamientos. En el alcance del presente trabajo nos enfocaremos a los costos por fallos internos, vinculados directamente con las acciones vencidas, del sistema de gestión de calidad, ya que los fallos externos en la organización objeto de estudio son clasificados como rechazos y su tratamiento es distinto.

De acuerdo con Valdizan (2016), la empresa objeto de estudio tiene tipificadas las acciones en:

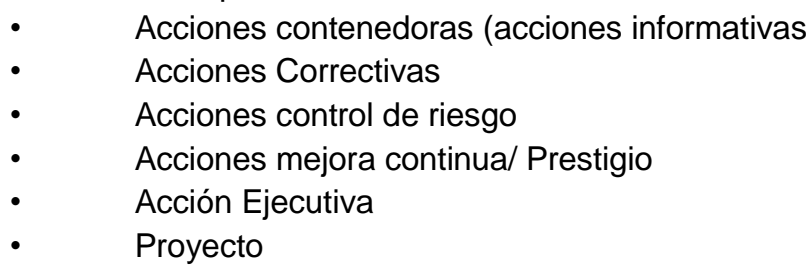

Las acciones correctivas deben ser apropiadas a los efectos de las no conformidades encontradas. (ISO, 2015). El sistema de gestión de calidad, ayuda a la organización, con el tratamiento de las acciones, evitando la recurrencia; sin embargo, para el caso de estudio, se ha detectado que el seguimiento de estas acciones no ha sido adecuado, dado que durante el periodo 2018 las acciones vencidas, se han visto incrementadas.

Al contextualizar estadísticamente los procesos organizacionales, se genera un referente para la toma de decisiones. Existe una serie de herramientas estadísticas vinculadas al control de calidad, las cuales afectan directamente los costos de la calidad, al crearse información que permite vislumbrar la situación que guarda la organización.

\section{Resultados}

Como ya se ha hecho mención, las acciones correctivas, de acuerdo al contexto de la empresa objeto de estudio, son aquellas tomadas para eliminar la causa raíz en una "no conformidad" y con lo cual se pretende evitar la recurrencia. A continuación, se visualizan las acciones correctivas (mensuales), para el periodo comprendido entre el mes de enero (2018) al mes de febrero (2019).

Tabla 1: Acciones vencidas y cerradas, para los periodos seleccionados. Fuente: Elaboración propia con base en la observación del proceso

\begin{tabular}{|l|c|c|c|}
\hline Mes & Año & $\begin{array}{c}\text { Número } \\
\text { de } \\
\text { acciones } \\
\text { vencidas }\end{array}$ & $\begin{array}{c}\text { Número } \\
\text { de } \\
\text { acciones } \\
\text { cerradas }\end{array}$ \\
\hline Enero & 2018 & 3 & 9 \\
\hline Febrero & 2018 & 1 & 5 \\
\hline Marzo & 2018 & 3 & 6 \\
\hline Abril & 2018 & 3 & 11 \\
\hline Mayo & 2018 & 11 & 17 \\
\hline
\end{tabular}




\begin{tabular}{|l|l|l|c|}
\hline Junio & 2018 & 13 & 24 \\
\hline Julio & 2018 & 28 & 33 \\
\hline Agosto & 2018 & 38 & 47 \\
\hline Septiembre & 2018 & 46 & 65 \\
\hline Octubre & 2018 & 31 & 67 \\
\hline Noviembre & 2018 & 41 & 87 \\
\hline Diciembre & 2018 & 27 & 30 \\
\hline Enero & 2019 & 37 & $\begin{array}{c}\text { No } \\
\text { disponible }\end{array}$ \\
\hline Febrero & 2019 & 48 & $\begin{array}{c}\text { No } \\
\text { disponible }\end{array}$ \\
\hline
\end{tabular}

A continuación, se realiza una caracterización básica de los datos vinculados al número de acciones vencidas (Tabla 2):

Tabla 1: Tabla clase-hoja-frecuencia. Fuente elaboración propia, con base en la caracterización de los datos

\begin{tabular}{|c|c|c|c|c|c|}
\hline Clase & $\frac{\frac{\pi}{0}}{\frac{1}{2}}$ & 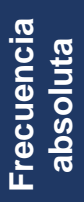 & 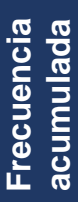 & 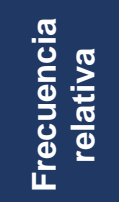 & 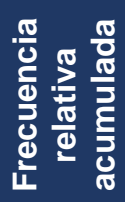 \\
\hline 01-09 & ||| & 4 & 4 & 0.333 & 0.333 \\
\hline $10-18$ & 11 & 2 & 6 & 0.167 & 0.500 \\
\hline $19-27$ & | & 1 & 7 & 0.083 & 0.583 \\
\hline $28-36$ & 11 & 2 & 9 & 0.167 & 0.750 \\
\hline $37-45$ & 11 & 2 & 11 & 0.167 & 0.917 \\
\hline \multirow[t]{2}{*}{$46-54$} & | & 1 & 12 & 0.083 & 1.000 \\
\hline & & $\Sigma=12$ & & $\Sigma=1.000$ & \\
\hline
\end{tabular}

Con base en los datos obtenidos al respecto de las acciones de calidad vencidas, se realizará el análisis estadístico, producto de la observación de la empresa objeto de estudio.

\subsection{Medidas de tendencia central}

Las medidas de tendencia central permiten observar la agrupación de los datos y su tendencia al valor esperado. Existen diferentes medidas de tendencia central, para el caso de la empresa objeto de estudio, se recurrirá a los estimadores para la media, mediana y moda.

Para el caso de la empresa objeto de estudio las medidas de tendencia central son:

$$
\begin{gathered}
\bar{x}=20.41666667 \text { acciones vencidas } \\
\text { mediana }=\widetilde{x}=20 \\
\text { moda }=3
\end{gathered}
$$

Dados los resultados anteriores, dos de las medias de tendencia central consideradas, indican que la propensión tiende a 20 acciones vencidas por mes, a pesar de que la moda indica, que el dato de mayor presencia en los registros es de 3 acciones vencidas (para los meses de enero, marzo y abril).

\subsection{Medidas de dispersión}

Por su parte, las medidas de dispersión permiten visualizar el acomodo de los datos, y su distribución en el eje de referencia. En este contexto, se tienen diferentes mediciones de dispersión de datos, como lo son: desviación estándar, varianza, rango, coeficiente de curtosis y coeficiente de asimetría, dichas mediciones son las más populares y usadas.

Para el caso de la empresa objeto de estudio, y con base en los registros por mes de la tabla 1, se tiene:

$$
\begin{gathered}
S_{X}=16.59933369 \text { acciones vencidas } \\
S_{X}^{2}=275.5378788 \\
\text { rango }=45 \text { acciones vencidas } \\
\text { asimetía }=0.200245351 \\
\text { curtosis }=-2.013224539
\end{gathered}
$$

De acuerdo con los cálculos anteriores de tiene un rango de datos igual a 45 acciones vencidas. Por otro lado, la desviación estándar es igual a 16.59933369 acciones vencidas, lo cual implica que el $65 \%$ de los datos se encuentra a una desviación estándar (hacia la izquierda y hacia la derecha) de la media, el $95 \%$ de los datos se distribuyen a dos desviaciones estándar y $99 \%$ de los datos se encuentran a tres desviaciones estándar.

El coeficiente de asimetría (asimetía $=\mathbf{0}$. 200245351), indica que existe asimetría positiva, por lo cual los datos tienden a agruparse a la izquierda de la media. En el caso del coeficiente de curtosis (curtosis $=\mathbf{- 2 . 0 1 3 2 2 4 5 3 9}$ ), la concentración de datos se distribuye de platocúrtica, por lo cual muestra una forma achatada.

\subsection{Coeficiente de correlación}

El índice de correlación mide la fuerza de interacción o dependencia existente entre dos variables. Para el cálculo de dicho índice se requiere calcular diferentes estimadores y momentos sobre las variables consideradas.

Para el caso de la empresa objeto de estudio, se utilizan como variables para el cálculo del índice de correlación, las acciones vencidas y cerradas para el periodo que comprende el año 2018 en sus diferentes meses. Como ya se hizo mención la el promedio para la variable $X$ es 20.416 ( $\overline{\boldsymbol{x}}=\mathbf{2 0 . 4 1 6 6 6 6 6 7}$ acciones vencidas), en tanto que para la variable $Y$ se tiene:

$$
\bar{y}=33.41666667 \text { acciones cerradas }
$$

La desviación estándar para la variable aleatoria $X$ esta $\begin{array}{llll}\text { dada } & \text { por } & 16.599 & \left(\boldsymbol{S}_{X}=\right.\end{array}$ 16.59933369 acciones vencidas), mientras que la desviación estándar para la variable $Y$ es:

$$
S_{Y}=27.31785408 \text { acciones cerradas }
$$

La covarianza entre las variables $X$ y $Y$, esta dada por:

$$
S_{X Y}=\operatorname{cov}(X, Y)=379.0763889
$$

Por lo tanto, el índice de correlación se expresa por:

$$
\rho_{X Y}=0.835967702
$$


Lo cual implica que existe un $83.59 \%$ de relación positiva entre las variables $\boldsymbol{X}$ y $\boldsymbol{Y}$, con base en ello se puede expresar que un cambio en la alguna de ellas, afecta directamente a la otra.

Al considerar las desviaciones estándar para la población, se tiene

$\rho_{X Y}=\frac{379.0763889}{(15.89265204)(26.15485402)}=0.911964766$ Que es el resultado que arrojado por formula COEF.DE.CORREL(matriz1;matriz2), de Microsoft ${ }^{\circledR}$ Excel® y la función de correlación (menú "estadística", submenú "estadísticas básicas", función "correlación") del programa Minitab® .

Lo cual indica una correlación de $91.19 \%$. Sin embargo, dado que los datos históricos usados en el presente estudio, corresponden a la muestra anual para las acciones vencidas y acciones cerradas, por ello se recomienda el uso de las ecuaciones (5) y (11) para cálculo de desviaciones estándar, aunadas a la ecuación (12) para el cálculo de la correlación estadística entre las variables.

\subsection{Regresión lineal (MCO)}

La regresión lineal, también conocida como mínimos cuadrados ordinarios, son una herramienta útil para el cálculo del pronóstico del periodo $\boldsymbol{t}+\mathbf{1}$, con base en la información histórica. Cabe hacer mención que, para esta sección, las variables serán tratadas de una forma que se adecue a la necesidad del pronóstico. Para nuestro caso, la variable aleatoria $\boldsymbol{Y}$ se considerará como las acciones vencidas para el mes dado, en tanto que la variable $\boldsymbol{X}$, se tomará como el número de periodo, vinculado al mes de referencia, de tal modo que el mes de enero del año 2018, se toma como el periodo cero (por conveniencia), en cuyo caso el mes de enero del año 2019 (periodo 12), será el dato que posibilita el pronóstico de las acciones vencidas, para dicho mes (Tabla 3).

Tabla 2: Adecuación de las variables, para aplicación del método de mínimos cuadrados. Fuente: Elaboración propia, con base en los datos recopilados del proceso

\begin{tabular}{|l|c|c|c|}
\hline Mes & Año & $\begin{array}{c}\text { X } \\
\text { [Número } \\
\text { de } \\
\text { periodo] }\end{array}$ & $\begin{array}{c}\text { Y } \\
\text { [Número de } \\
\text { acciones } \\
\text { vencidas] }\end{array}$ \\
\hline Enero & 2018 & 0 & 3 \\
\hline Febrero & 2018 & 1 & 1 \\
\hline Marzo & 2018 & 2 & 3 \\
\hline Abril & 2018 & 3 & 3 \\
\hline Mayo & 2018 & 4 & 11 \\
\hline Junio & 2018 & 5 & 13 \\
\hline Julio & 2018 & 6 & 28 \\
\hline
\end{tabular}

\begin{tabular}{|l|c|c|c|}
\hline Agosto & 2018 & 7 & 38 \\
\hline Septiembre & 2018 & 8 & 46 \\
\hline Octubre & 2018 & 9 & 31 \\
\hline Noviembre & 2018 & 10 & 41 \\
\hline Diciembre & 2018 & 11 & 27 \\
\hline
\end{tabular}

Con base en las diferentes ecuaciones para el método de mínimos cuadrados ordinarios, se tiene:

a su vez,

$$
b=\frac{47.125}{11.91666667}=3.9545455
$$

$$
\begin{gathered}
a=20.41666667 \\
=-3.9545455 *(5.5)
\end{gathered}
$$

con la estimación de los parámetros $\boldsymbol{a}$ y $\boldsymbol{b}$, la recta de ajuste es:

$$
\widehat{Y}=-1.33333333+3.9545455 * X
$$

Es decir, el pronóstico para el periodo 12 (enero del año 2019) es:

$$
\begin{aligned}
& \widehat{\boldsymbol{y}}_{t+1}=\widehat{\boldsymbol{y}}_{12}=-1.33333333+3.9545455 *(12) \\
&= 46.12121212 \text { Acciones vencidas }
\end{aligned}
$$

Lo cual indica que para el periodo $t+1$ se espera que se presenten alrededor de 46 acciones vencidas.

Para nuestro estudio la variación explicada está dada por:

$$
\begin{aligned}
V E=(-1.333333333-20.41666667)^{2}+\cdots \\
+ \\
+(42.16666667-20.41666667)^{2} \\
=2236.295455
\end{aligned}
$$

En tanto que la variación no explicada tiene como resultado:

$$
\begin{aligned}
& V N E=(3-(-1.333333333))^{2}+\ldots+(27 \\
& -42.1666667)^{2}=794.6212121
\end{aligned}
$$

Por lo cual, la variación total, es:

$$
\begin{gathered}
V T=(3-20.41666667)^{2}+\cdots+(27-20.41666667)^{2} \\
=3030.916667
\end{gathered}
$$

Con base en los cálculos anteriores, el coeficiente de determinación es:

$$
\begin{gathered}
R^{2}=1-\frac{794.6212121}{3030.916667}=1-0.26217191 \\
=0.73782809
\end{gathered}
$$

O en su caso:

$$
R^{2}=\frac{2236.295455}{3030.916667}=0.73782809
$$

Lo cual indica que la recta de ajuste, explica un $73.78 \%$ a los datos de la variable de referencia. Mediante el programa Minitab ${ }^{\circledR}$ se puede corroborar los resultados obtenidos, tanto para la recta de ajuste, como para el coeficiente de determinación.

\section{Conclusión}

Dentro de una organización se presentan diversos problemas y la manera dar solución a ellos, es mediante la continuidad a las acciones dentro del sistema organizacional y en particular dentro del sistema de 
gestión de calidad. De no ser así, los problemas tienden a incrementarse, dando a entender que la organización no está tiene una vigilancia y seguimiento del comportamiento correspondiente a las acciones.

Los costos de calidad dentro del sistema de gestión de la calidad (SGC) son un área prioritaria al interior de las organizaciones, ya que, como instrumento de gestión, permite identificar todo aquello relacionado con el producto $y / o$ servicio ofertado al cliente final. El cuidado del cumplimiento en la entrega de acciones de calidad, permite apoyar el correcto desempeño del sistema de gestión de calidad en las empresas, con lo cual se tiene una propensión a la reducción de costos asociados a la mala calidad.

El uso de herramientas estadísticas, permite a las organizaciones, generar información que posibilite la toma de decisiones sobre el sistema de gestión de calidad. Lo anterior, da la pauta para el control y reducción de acciones vencidas, con lo que se pueden incrementar los niveles de calidad en la organización y, por ende, la satisfacción del cliente o consumidor final.

\section{Referencias}

Arredondo-González, M. M. (2015). Contabilidad y análisis de costos. (Primera ed.). Ciudad de México, México: Grupo Editorial Patria.

Gómez-Alfonso, E. (2013). Cálculo de los costos de calidad en la Unidad Empresarial de Base Producciones Varias, Cienfuegos, Visión de Futuro, Año 10, 17(2), consultado de:

[https://dialnet.unirioja.es/descarga/articulo/5234058.pdf].

ISO 9001:2015. (2015). Sistemas de gestión de la calidad-Requisitos. En ISO. Ginebra- Suiza.

Valdizan, M. (2016). Acciones correctivas, preventivas y de mejora continua. NC204-A1, 11.

Wang, T., Chen, Y., Qiao, M., \& Snoussi, H. (2018). A fast and robust convolutional neural network-based defect detection model in product quality control. The International Journal of Advanced Manufacturing Technology, 94(9-12), 3465-3471, DOI: 10.1007/s00170-017-0882-0, consultado de: [https://link.springer.com/content/pdf/10.1007/s00170017-0882-0.pdf]. 